\title{
Determinantes del precio del suelo como variable proxy de las preferencias idiosincráticas de los individuos en un marco teórico de equilibrio general ${ }^{\star}$
}

\section{Determinants of land prices as proxy variable of idiosyncratic preferences of individuals in a general equilibrium theoretical framework}

Recibido: 11 de octubre de 2014

Revisado: 15 de noviembre de 2014

Aprobado: 16 de marzo de 2015

\section{Resumen}

Los fenómenos urbanos se caracterizan desde diferentes vertientes teóricas, por ejemplo, el equilibrio general espacial que modela el comportamiento de consumidores, firmas e impactos en la movilidad por la densificación o distribución aleatoria de los agentes en el territorio, que constituye el problema del planeador centralizado del territorio. Sin embargo, la presencia de elementos inobservables de las preferencias o gustos

* Resultado de la tesis de maestría "Análisis empírico en el precio del suelo. Localidad de Puente Aranda, periodo 2000 /2010". Como citar este articulo: Talero, J. (2014). Determinantes del precio del suelo como variable proxy de las preferencias idiosincráticas de los individuos en un marco teórico de equilibrio general. Revista CIFE, 16 (25), $17-41$.

** Magíster en Ciencias Económicas de la Universidad Nacional de Colombia. Artículo resumen de los principales resultados de la investigación para aspirar al título. Correo electrónico: jatalerob@unal.edu.co 
idiosincráticos de los agentes dueños del territorio usualmente no es modelable, pero constituye un bloqueo para la renovación urbana. En este artículo se repasan algunos de los principales supuestos de un modelo de equilibrio general y su validez empírica en términos de las dinámicas de precios, para finalmente plantear una forma de modelar aquellas preferencias idiosincráticas inobservables en los cambios de un territorio, utilizando la técnica de datos panel.

Palabras clave: renovación urbana, localización industrial, preferencias idiosincráticas, precios del suelo.

Clasificación JEL: D12, D24, D58, R13, R30, R32

\section{Abstract}

Urban phenomena are characterized from different theoretical aspects, for example, spatial general equilibrium modeling the behavior of consumers, firms and impacts on mobility densification or random distribution of agents in the territory, which is the problem of the centralized planner of the territory. However, the presence of unobservable elements like idiosyncratic preferences or tastes of the territory owners usually are not moldable, but they are a lock for urban renewal. This article reviews some of the main assumptions of a general equilibrium model and its empirical validity in terms of price dynamics, to finally raise a way to model those unobservable idiosyncratic preferences, using the panel data technique.

Keywords: Urban Renewal, industrial location, land Price, idiosyncratic preferences.

Classification JEL: D12, D24, D58, R13, R30, R32 


\section{Introducción}

El estudio de las ciudades tradicionalmente se centra en analizar asentamientos monocéntricos y policéntricos, esto es, una visión de la perspectiva de la centralidad de una actividad económica que conlleva a un desarrollo de equipamientos, localización de agentes y necesidades de espacio público, atendiendo una dinámica económica de oferta y demanda de productos y servicios. Pero las dinámicas económicas y el paso del tiempo generan presiones sobre los equipamientos presentes en las centralidades, y surgen necesidades de renovación urbana que usualmente constituyen procesos conflictivos entre las necesidades de espacio público versus las preferencias de los agentes locales.

En los procesos de formulación de renovación urbana generalmente no se modela el impacto de las preferencias de los agentes locales, lo que constituye, en la práctica, bloqueo a la actividad de renovación. El presente artículo busca demostrar cómo los agentes exhiben ciertas preferencias idiosincráticas que implican afectación de los precios del suelo para orientar una discusión más profunda sobre las necesidades de incluir las preferencias de los agentes en la formulación de un modelo de renovación urbana.

El artículo está basado en un contexto teórico relacionado con ciertas condiciones de equilibrio iniciales, bajo algunos supuestos y el enfoque del modelo original de AnasKim $(1996)^{1}$ para un análisis ortodoxo, apoyándose en información empírica obtenida de una base de datos de predios, destinos y usos de los años 2000 y 2010, además de la información de los registros catastrales y el Registro Mercantil de 2010.

Se estimaron tres elementos: a) Modelo de Efectos Fijos, que permitió concluir que a mayor densidad de los equipamientos hay mayor avalúo, que las propiedades particulares en general son más avaluadas que las propiedades oficiales y que el espacio público, y finalmente, que los destinos residencial e industrial generan menor precio frente a destinos como terrenos urbanizables no urbanizados (o lotes de terreno); b) Modelo de Efectos Aleatorios para identificar el comportamiento no observable de los residentes o la presencia de preferencias idiosincráticas para mantener un uso específico del suelo, lo cual fue comprobado a partir de la significancia estadística del modelo y manteniendo las conclusiones del Modelo de Efectos Fijos; y c) rendimientos a escala de las firmas, identificando que en la localidad se ubica un importante número de firmas con tecnología asimilada a rendimientos constantes a escala.

Los resultados prueban que, dada la presencia de las preferencias idiosincráticas, es importante garantizar sostenibilidad en las políticas públicas desde las necesidades de mayor densificación, renovación y urbanización, en coherencia con instrumentos

1 El documento original "General equilibrium models of polycentric urban land use with endogenous congestion and job agglomeration" fue publicado en 1996. 
orientados a normalizar los precios del suelo para compensar las diferencias entre los avalúos de los distintos propietarios del suelo y su destino.

El artículo se organiza de la siguiente manera. La primera sección corresponde a la introducción; la segunda parte presenta el marco teórico conceptual; el tercer acápite explica la metodología, describe los datos disponibles para el análisis y expone comentarios de los resultados; y en la cuarta sección se realiza una discusión sobre las conclusiones.

\section{Marco teórico}

\subsection{Tendencias en el análisis de la renovación urbana}

La literatura de las ciudades es abundante y enriquecida por distintas vertientes teóricas; como Santagata y Ortona (1981) lo señalan, la búsqueda de la explicación de las dinámicas de asentamiento de usos residenciales e industriales y su movilidad en diferentes espacios geográficos puede ser considerada como una vertiente de una teoría general, siendo entonces un componente del fenómeno de crecimiento o la disminución de la actividad industrial en el ámbito metropolitano. Se puede utilizar entonces como instrumento el equilibrio general; por ejemplo, el Modelo de Anas-Kim (1996) relaciona tres líneas de investigación independientes:

a. Modelos de equilibrio parcial con ciudades que presentan dos centros de trabajo cuya localización está predefinida.

b. Modelos que no especifican centros de localización del trabajo, pero develan la densificación y la aglomeración de actividades alrededor del espacio más accesible, con impactos sobre la renta del suelo.

c. Modelos que explican la formación de una aglomeración endógena por la búsqueda de la maximización de beneficios dado el aprovechamiento de externalidades positivas derivadas de la co-localización de agentes económicos y los costos que se presentan por interactuar con agentes de otra localización.

Este enfoque teórico constituye un modelo de equilibrio general computable de usos del suelo determinados desde la localización de los hogares, las actividades productivas y los equipamientos de movilidad (caminos y carreteras), en el cual se conjugan elementos como la congestión de tráfico y la localización del empleo como variables endógenas; los consumidores eligen el trabajo y su lugar de residencia, además, su comportamiento implica que consumirán bienes finales en cualquier zona del espacio.

El consumo no presenta economías de escala, por lo tanto, la producción se distribuye entre rentas, salarios y precios de los insumos. Las preferencias de los consumidores 
expresan que, entre mayor aglomeración de comercio, mayor voluntad por realizar actividades de consumo, dando origen a centralidades económicas.

Dada la variable de congestión de tráfico, es posible que se generen dispersiones inestables de localización de la oferta (la actividad productiva), así surgen múltiples equilibrios alrededor de múltiples centros, siendo los choques teóricos de este modelo, procesos de transición de la aglomeración de la actividad productiva entre los diferentes centros en un trade-off entre los beneficios derivados por la aglomeración frente a los costos de acceso a las centralidades.

\subsection{El problema del consumidor racional}

Cada consumidor toma como dados la distribución del empleo, la localización de los centros de comercio, el tiempo de transporte y los costos asociados a la movilidad, además, son precio-aceptantes en todos los mercados.

Lo que determina el problema del consumidor es la localización del trabajo y la ubicación de su residencia (variables $(i, j)$ respectivamente), el tamaño de la residencia o del lote del terreno que usará, las horas laborales y de ocio, y la función de viajes para adquirir bienes y servicios.

Este problema se resuelve en dos etapas. La primera consiste en el problema de maximización de utilidad, donde la función que se asumirá será de tipo Cobb Douglas homogénea de grado $1^{2}$, y que tendrá la siguiente forma:

$U_{i j}=\sum_{r k} \alpha_{r k} \ln Z_{i j r k}+\beta \ln q_{i j}+\gamma \ln L_{i j}+u_{i j}$ donde:

$Z_{i j r k}$ Es la cantidad del r-ésimo bien comprado en la zona de producción $k$ a un precio unitario $p_{r k}$.

$q_{i j}$ es el tamaño del lote del consumidor en la zona de residencia $i$.

$L_{i j}$ es el tiempo de ocio del consumidor.

$u_{i j}$ son constantes de idiosincrasia individual.

La restricción presupuestaria en este problema está definida a partir de las siguientes variables:

2 Por simplicidad, en este modelo se asume que los parámetros $\alpha_{r k}, \beta, \gamma$ son idénticos entre los consumidores, y que, además, $\Sigma_{r k} \alpha_{r k}+\beta+\gamma=1$. 
$\rho_{i}$ es la renta del terreno en la zona residencial $i$.

$w_{j}$ es el salario por horas laboradas en la zona productiva $j$.

$H$ corresponde a las horas totales disponibles para trabajar, descansar y viajar por periodo.

$v_{t i j}$ son los días laborables al año.

$t_{i k}$ es el costo monetario de realizar un viaje de ida desde la zona residencial $i$ al centro de actividad económica $k$.

$T_{i j}$ es el tiempo total de viaje por periodo.

$g_{i k}$ es el tiempo de un viaje de ida para hacer compras, desde la zona residencial $i$ hasta la zona residencial $k$.

$2 v_{t i j}$ es el número de desplazamientos por periodo.

$D$ son los dividendos de la renta de la tierra.

Así, el problema del consumidor racional es:

$$
\max _{q_{i j}, L_{i j}, Z_{i j}} \sum_{r k} \alpha_{r k} \ln Z_{i j r k}+\beta \ln q_{i j}+\gamma \ln L_{i j}+u_{i j}
$$

Sujeto a:

$$
\sum_{r k} Z_{i j r k}\left(p_{r k}+2 t_{i k}\right)+\rho_{i} q_{i j}+2 v_{t i j}=w_{j}\left(H-T_{i j}-L_{i j}\right)+D
$$

En la ecuación, el ingreso del consumidor se determina por la expresión $w_{j} H+D$, la cual, con el consumo de los bienes de la economía, agota la tierra para adquirir su vivienda, el costo de sus desplazamientos y el uso de su tiempo de descanso.

De esta manera, las expresiones de ocio y desplazamientos se adquieren a un costo de oportunidad del momento, expresado por la tasa de salarios $w_{j}$, al trabajar menos y disminuir su ingreso potencial por el salario obtenido por cada hora laborada.

En esta condición, también el presupuesto se gasta en el costo completo de los viajes. Para un viaje de ida y vuelta se encuentra la expresión:

$$
p_{r k}+2 t_{i k}+2 w_{j} g_{i k}
$$


Y para un desplazamiento es $t_{i j}+w_{j} g_{i j}$.

El tiempo total de viaje se calcula de la siguiente manera:

$T_{i j}=2 v g_{i j}+\sum_{r k} 2 g_{i k} Z_{i j r k}$ donde $2 Z_{i j r k}$ es el número de viajes de ida para hacer compras.

La tierra está distribuida equitativamente en la economía, así, cada individuo es dotado de una propiedad igual, lo que hace que las rentas agregadas sean redistribuidas por el dividendo de tierra $D$, derivado del siguiente comportamiento:

Sean $\mathcal{N}$ el número exógeno de consumidores y $A_{\mathrm{i}}$ la cantidad de tierra total por distribuir en la zona residencial i, el dividendo de la renta de la tierra será:

$$
D=\frac{\sum_{i} A_{i} \rho_{i}}{N}
$$

El término $u_{i j}$ correspondiente a las constantes de idiosincrasia individual se refiere al único valor diferencial entre los consumidores ${ }^{3}$.

En la primera etapa de la maximización, como las variables $(i, j)$ son exógenas y están definidas, entonces $u_{i j}$ será una constante para cada consumidor ${ }^{4}$.

Para cada bien $r$ adquirido en la zona $k$, la cantidad demandada está definida por la siguiente función:

$$
* Z_{i j r k}=\alpha_{r k} \frac{w_{j} H-2 v\left(t_{i j}+w_{j} g_{i j}\right)+D}{\rho_{r k}+2 t_{i k}+2 w_{j} g_{i k}}
$$

En dicha función también se encuentra presente la cantidad demandada de viajes.

La cantidad de suelo para uso residencial está definida por la siguiente expresión:

$$
{ }^{*} q_{i j}=\beta \frac{w_{j} H-2 v\left(t_{i j}-w_{j} g_{i j}\right)+D}{\rho_{i}}
$$

3 A pesar de que es el único valor que diferencia las preferencias de los consumidores, realmente es el efecto que puede limitar procesos completos de renovación urbana o transformaciones del territorio; usualmente estos últimos no logran ser consistentes con las preferencias individuales.

4 Limitando la evidencia empírica, como esta variable es inobservable, nada garantiza que estas preferencias individuales idiosincráticas se encuentren caracterizadas en la solución de equilibrio, preferencias que sí tienen relevancia para el comportamiento de los asentamientos y usos del suelo, como se mostrará en los resultados. 
El ocio demandado es:

$$
* L_{i j}=\gamma \frac{w_{j} H-2 v\left(t_{i j}-w_{j} g_{i j}\right)+D}{w_{j}}
$$

La función de utilidad indirecta será:

$$
U_{i j}=\ln \left[w_{j} H-2 v\left(t_{i j}+w_{j} g_{i j}\right)+D\right]-\sum_{r k} \alpha_{r k} \ln \left(\rho_{r k}+2 t_{i k}+2 w_{j} g_{i k}\right)-\beta \ln \rho_{i}-\gamma \ln w_{j}+u_{i j}
$$

Esta es, entonces, la máxima utilidad posible, dada la elección del consumidor referente a su elección de ubicación de trabajo y residencia $(i, j)$.

Pero esta elección se deriva de una segunda maximización del consumidor en un problema definido a partir de la comparación de las utilidades indirectas de cada consumidor. $\mathrm{Al}$ permanecer el parámetro $u_{i j}$, o gusto idiosincrásico para elegir el mejor lugar de residencia y de trabajo, entonces las variables $(i, j)$ variarán de acuerdo con dicho gusto, haciendo que la elección sea diferente para todos los consumidores, por lo tanto, la distribución será aleatoria en el territorio ${ }^{5}$.

\subsection{El problema de la firma}

Dada la localización donde operan, las firmas son tomadoras de precio de los productos y de los insumos, además, toman los costos de transporte de sus bienes intermedios como dados.

Lo que decidirá cada firma es la demanda por trabajo, cantidad de tierra como factor productivo y la demanda de bienes intermedios requeridos para su actividad, de otras localizaciones. El modelo asume que los bienes intermedios de características similares producidos en un lugar diferente son efectivamente bienes distintos ${ }^{6}$.

\section{La tecnología tendrá rendimientos constantes a escala, por lo tanto, el máximo beneficio es cero ${ }^{7}$.}

Por lo anterior, una firma producirá el bien $r$ en la ubicación $j$ a partir de una tecnología de tipo Cobb Douglas. La variable $X_{r j}$ es la producción industrial, $M_{r j}$ es el factor productivo del empleo, $Q_{r j}$ es el factor productivo tierra y $Y_{r j s n}$ es la cantidad del bien s necesario de la locación $n$, usado como bien intermedio.

\footnotetext{
5 A partir de la información disponible, se utilizará la técnica de datos panel, identificando efectos fijos y efectos aleatorios.

6 Supuesto principal de una economía Arrow - Debreu (1954).

7 Cuya demostración se puede representar a partir del teorema de Euler.
} 
Determinantes del precio del suelo como variable proxy de las preferencias idiosincráticas de los individuos...

La función de producción es:

$$
X_{r j}=M_{r j}^{\delta_{r}} Q_{r j}^{\mu} \prod_{s n} Y_{r j s n}^{\phi_{r s n}}
$$

Por rendimientos constantes a escala, $\delta_{r}+\mu_{r}+\sum_{s n} \phi_{r s n}=1$.

Entendiendo a la firma como maximizadora de beneficios, el problema de racionalidad se transcribe de la siguiente manera:

$$
\max _{\left[m_{r j}, Q_{r j}, Y_{r j}\right]} \pi_{r j}=p_{r j} X_{r j}-M_{r j} w_{j}-Q_{r j} \rho_{j}-\sum_{s n} Y_{r j s n}\left(p_{s n}+m_{s} t_{n j}\right)
$$

En la ecuación, m es la cantidad equivalente de pasajeros de una unidad de carga.

Las demandas de insumos, condicionales en el ámbito de producción y que resuelven el problema de maximización, están determinadas por la propiedad de los coeficientes de la función de producción y son las participaciones en los costos del insumo correspondiente:

$$
\begin{gathered}
M_{r j}=\delta_{r} p_{r j} w_{j}^{-1} X_{r j} \\
Q_{r j}=\mu_{r j} p_{r j} \rho_{j}^{-1} X_{r j} \\
Y_{r j s n}=\phi_{r s n} p_{r j}\left(p_{s n}+m_{s} t_{n j}\right)^{-1} X_{r j}
\end{gathered}
$$

Dados los rendimientos constantes a escala, los costos se incrementan linealmente en proporción a la producción ${ }^{8}$.

Dada la propiedad de máximo beneficio cero a cualquier escala de producción, el precio del producto se puede expresar en función del precio de los insumos, incluyendo el precio propio, ya que los bienes intermedios pueden ser usados como insumos en su propia producción:

$$
p_{r j}=\frac{w_{j}^{\delta_{r}} \rho_{j}^{\mu_{r}} \Pi_{s n}\left(p_{s n}+m_{s} t_{n j}\right)^{\phi_{r s n}}}{\delta_{r}^{\delta_{r}} \mu_{r}^{\mu_{r}} \prod_{s n} \phi_{r s n}^{\phi_{r s n}}}
$$

\subsection{El equilibrio general competitivo}

Si asumimos que $I$ zonas urbanas se dividen en rectángulos de poca anchura y con una unidad de longitud específica ${ }^{9}$, y que existe un número finito de $\mathcal{N}$ consumidores, las

8 Por la propiedad de homogeneidad de grado 1 de la función de producción, el costo marginal y el costo medio son equivalentes y constantes.

9 La ciudad linear, de Solow and Vickery, referenciado en Anas Kim, p. 11 
dotaciones iniciales de tiempo disponible $H$ y la totalidad de la tierra $A_{i}$ se encontrarán distribuidas entre $\operatorname{los} \mathcal{N}$ consumidores.

El modelo encontrará los precios para cada bien, por zona y por tipo de bien $p$, las rentas de la tierra $r$, los salarios por zona $w$, la producción por zona de cada tipo de bien $X$ y los precios de los peajes de congestión de equilibrio $t$ junto con los tiempos de viaje por zona $g$.

Se satisface que la suma de los lotes para residencias demandados por todos los hogares que residan en la zona $i$, que viajan a todas las zonas, más la demanda por tierra de todas las firmas en la zona $i$ más la tierra utilizada para avenidas, caminos o carreteras sea igual a la tierra disponible en la zona $i$.

Esto es:

$$
N \sum_{j} \psi_{i j}(p, \rho, w) q_{i j}\left(w_{j}, \rho_{i}\right)+\sum_{r} Q_{r i}\left(p_{r i}, \rho_{i}, X_{r i}\right)+S_{i}=A_{i}
$$

Una segunda condición relaciona la oferta de trabajo de los consumidores que laboran en la zona $i$, que equivale a la demanda por trabajo de todas las firmas que se ubican en la zona $i$ :

$$
N \sum_{j} \psi_{i j}(p, \rho, w)\left[H-T_{s i}-L_{s i}\left(w_{i}\right)\right]=\sum_{r} M_{r i}\left(p_{r i}, w_{i}, X_{r i}\right)
$$

El tiempo total de viaje, representado por la ecuación $T_{i j}=2 v g_{i j}+\sum_{r k} 2 g_{i k} Z_{i j r k}$, está dado por el equilibrio parcial del sector de transporte. En el mercado por el bien $(r, i)$ se cumple que la cantidad de mercancía $r$ adquirida en $i$ por los consumidores que trabajan y viven en todas las parejas de zonas $(i, j)$ más la demanda del mismo bien, para ser utilizada como insumo intermedio por las firmas que producen en todas las zonas, es equivalente a la producción industrial de r-ésimo producto en la zona $i$ :

$$
N \sum_{n s} \psi_{n s}(p, \rho, w) Z_{n s i r}\left(w_{s}, p_{r i}\right)+\sum_{n s} Y_{n s i i}\left(p_{n s}, p_{r i}, X_{n s}\right)=X_{r i}
$$

La última condición es la relación derivada de la combinación entre la producción de bienes, los precios de los insumos, implicada por la condición de máximo beneficio cero para todas las firmas que producen el bien r-ésimo en la zona $i$.

$$
p_{r i}-p_{r i}\left(w_{i}, \rho_{i}, p\right)=0
$$

La cantidad de ecuaciones por resolver es $2 I+2 R I$, y las variables por resolver son:

$$
p, X, w, \rho
$$


Determinantes del precio del suelo como variable proxy de las preferencias idiosincráticas de los individuos...

Las soluciones del sistema de equilibrio general serán homogéneas de grado cero en todos los precios $p$, rentas $r$ y salarios $w$, lo que implica que los precios se podrán normalizar y las demandas se podrán expresar en términos de precios relativos.

\section{Hipótesis y evidencia empírica}

Dado que, en los modelos de equilibrio general, la solución competitiva está definida por las tres etapas o modelos del consumidor, la firma y el sector público, las hipótesis fundamentales son las siguientes:

a. La existencia de variables inobservables del comportamiento de los dueños de las tierras de un territorio específico es significativa a la hora de fijar los precios del suelo. Estas variables se denominan preferencias idiosincráticas, por lo tanto, su modelización es relevante para la definición del impacto de una actuación de renovación urbana.

b. La sensibilidad del precio del suelo también puede caracterizarse a partir de las presiones de densificación de las zonas. La relación de densificación tiene impactos sobre los avalúos de los territorios, siendo significativos en el sentido estadístico. Este resultado permitirá identificar las dinámicas de la fijación de precio en la localidad.

Los resultados de las estimaciones comparativos se observan en la tabla 1:

Tabla 1. Comparativo de resultados estimación por efectos fijos vs. efectos aleatorios 2000 / 2010

\begin{tabular}{|l|r|r|r|r|}
\hline \multicolumn{3}{|c|}{ Variable endógena: avalúo } \\
\hline Variables exógenas & Efectos fijos & Prob. t & Efectos aleatorios & Prob. t \\
\hline Densidad & 51.700 .000 & 0,000 & 3.218 .372 & 0,000 \\
\hline Tipo de propiedad & $(34.500 .000)$ & 0,000 & $(19.200 .000)$ & 0,000 \\
\hline Código de destino & 21.600 .000 & 0,000 & 3.879 .722 & 0,002 \\
\hline Área del terreno & 25.821 & 0,000 & 291.603 & 0,000 \\
\hline Área construida & 24.500 .000 & 0,010 & 54.800 .000 & 0,000 \\
\hline Coeficiente de determinación & 0,11 & & 0,5574 & 0,000 \\
\hline Probabilidad valor F & 1 & & & \\
\hline
\end{tabular}

Fuente: cálculos del autor. 


\subsection{Interpretación estimación de efectos fijos}

En esta estimación se evidenció que las variables densidad y tipo de propiedad están relacionadas con el precio del avalúo, pasando las pruebas t individual para cada estimador y la prueba de significancia estadística conjunta.

Dadas unas agrupaciones realizadas con anterioridad para mejorar la estimación (ver anexos, corresponde a variables categóricas al tipo de propiedad y al destino o uso del suelo), la interpretación de los coeficientes que explican las dinámicas de los avalúos se comportan de la siguiente manera:

Mayor densidad, mayor avalúo. En la medida en que se permita mayor factor de densificación, el avalúo -que es la sumatoria del precio del terreno y el precio de la construcción- aumentará.

Coeficiente tipo de propiedad. Las propiedades particulares (variable categórica 0) tienen mayor avalúo que las propiedades oficiales (cuya variable categórica 1) y, a su vez, estas tienen mayor avalúo que las destinadas a espacio público (variable categórica 3).

Coeficiente destino. Los destinos diferentes al uso residencial (variable categórica 0) y al uso industrial (variable categórica 1) presentarán mayor precio (en general, corresponden a lotes de terreno no urbanizados, pero que no son reservas ambientales).

Los resultados anteriores resaltan que es importante garantizar sostenibilidad en las políticas públicas, con mayor densificación, mitigando el impacto de una actuación de política en los precios a partir de algún instrumento de intervención en los precios del terreno, que compense las diferencias entre los avalúos de los distintos propietarios del suelo.

Mayor área de terreno, mayor avalúo. Resultado esperado en la medida en que el lote sea más grande. Su valor por metro cuadrado aumenta, por ende, el valor del avalúo se incrementa.

Mayor área de construcción, mayor avalúo. Resultado esperado en la medida en que el área construida aumenta. Su valor por metro cuadrado asciende, por consiguiente, el valor del avalúo se incrementa.

Nótese que la densidad incluye un cálculo sobre área de terreno y área construida; el efecto puede presentar multicolinealidad entre los dos estimadores, ya que la densidad está relacionada directamente con el área construida e inversamente con el área del lote.

Dado que se observa una probabilidad valor $F=1$, se debe realizar la estimación de efectos aleatorios para identificar los efectos del comportamiento no observable de los residentes ${ }^{10}$.

10 Variable idiosincrática que representa las preferencias para mantener un uso específico del suelo, en particular, el residencial. 


\subsection{Interpretación de la estimación de efectos aleatorios}

En los signos de los coeficientes, los resultados son equiparables a los efectos fijos. El área construida tiene un mayor coeficiente sobre el valor del avalúo, lo mismo ocurre con el área de terreno y la densidad que los relaciona a los dos.

El tipo de propiedad mantiene su relación inversa ${ }^{11}$, lo cual quiere decir que, en efecto, se tiende a apreciar con mayor importancia el terreno cuyo dueño es un particular, frente a los terrenos que son propiedades oficiales y aquellos que son de espacio público.

El resultado en los usos del terreno señala que se aprecia más un destino diferente al uso residencial (variable categórica 0) y al uso industrial (variable categórica 1); estos son usos como urbanizables no urbanizados (lotes de terreno dentro de la localidad), o los usos dotacionales como recreacional, institucional, recreacional, dotacional, lote del estado, vía pública, conservado indefinido.

La productividad de las firmas, determinada por su limitante tecnológica, es observable en un sentido empírico, deberá ser una función significativa sobre las características del territorio donde la actividad productiva se localice, permitirá validar el hecho del comportamiento del sector productivo con rendimientos constantes a escala en un modelo de equilibrio general.

\subsection{La función de producción de las firmas}

Asumiendo la tecnología de tipo Cobb Dougla en un modelo log - log, se estimó la siguiente función a partir de la técnica de los mínimos cuadrados ordinarios:

$$
y=A+\alpha \ln a+\beta \ln e+\delta \ln t
$$

En la ecuación:

$A=$ constante de tecnología

$a=$ activos o medida de la inversión en bienes de capital de cada firma

$e=$ empleo generado por cada firma

$t=$ área de terreno utilizada por cada firma.

Como resultado se obtuvo la siguiente ecuación:

$$
y=14,82+0,171270 \ln a+0,762328 \ln e+0,043526 \ln t
$$

11 Como el coeficiente del tipo de propietario del suelo mantiene su relación inversa, el resultado señalaba que las propiedades particulares $($ dummy $=0)$ tienen mayor avalúo que las propiedades oficiales $($ dummy $=1)$ y, a su vez, estas tienen mayor avalúo que los terrenos destinados a espacio público (dummy $=3$ ). 
ISSN: 0124-3551 / Año 16, No 25 / julio-diciembre / pp. 17-41

(0.0838) (0.0057) (0.0175) (0.0104) Desviación estándar

(0.0001) (0.0007) (0.0005) (0.0004) Probabilidad individual

Con un coeficiente de determinación de 0.51 y un estadístico F lo suficientemente grande que minimiza la probabilidad de cometer un error tipo I (prueba de significancia conjunta), al sumar los coeficientes $\alpha+\beta+\delta=0,97$ se ha probado empíricamente que las firmas presentes en la localidad tienden a satisfacer la homogeneidad de grado 1 o rendimientos constantes a escala, por tanto, su comportamiento puede asumirse cercano al establecido en el contenido teórico del equilibrio general.

\section{Conclusiones}

Existe evidencia empírica de que las preferencias idiosincráticas referenciadas en los modelos teóricos de equilibrio general exhibidos en la presente investigación existen en la localidad; además, sus impactos son significativos en términos de la modelación de actuaciones de renovación urbana por su impacto en los precios del suelo.

También se demostró que los precios del suelo tienen especial sensibilidad sobre los factores de densificación. En la medida en que el uso potencial del territorio sea más flexible, es decir, que se favorezca la edificabilidad, la actividad industrial podría tener impactos positivos en su productividad. Dichos factores deben analizarse en el sentido de la movilidad, la necesidad de espacios públicos y la capacidad de anticipación de cambios de los precios del territorio, en una autoridad pública o en un proyecto urbanista privado.

Se encontró que los usos o destinos y los tipos de propietarios de los inmuebles impactan sobre el nivel general de precios de cada uno, más aún, la teoría de equilibrio general no contempla una serie de características del territorio que enriquecerían el análisis, por ejemplo, los precios hedónicos; es posible que esas características favorezcan la modelación y permitan superar precisamente las limitantes en la formulación de un proyecto de renovación urbana frente a las preferencias idiosincráticas de las comunidades residentes.

En las características del territorio se puede incluir la caracterización de las firmas que realizan actividades productivas; en este sentido, los impactos locales de su producción o comercio y la tendencia observada de rendimientos constantes a escala favorecen el hecho de la modelización a partir del equilibrio general como instrumento, complementado con los análisis de los precios hedónicos y sus impactos sobre la interacción con las comunidades residentes. El análisis puede ampliarse para una zona geográfica mayor. 
Determinantes del precio del suelo como variable proxy de las preferencias idiosincráticas de los individuos... Jorge Andrés Talero Bernal

\section{Referencias}

Anas, A., \& Kim, I. (1996). General equilibrium models of polycentric urban land use with endogenous congestion and job agglomeration. Fournal of Urban Economics, 40(2), 232-256. Recuperado de http://goo.gl/1ROGdl

Araque, A. y Cubillos, R. (2001). Los precios del suelo urbano en Santa Fe de Bogotá 2001-2010: Las plusvalias después del POT. Bogotá: Departamento Administrativo de Planeación Distrital.

Arrow, K. J., \& Debreu, G. (1954). The existence of an equilibrium for a competitive economy. Econometrica, 22(3), 265-290.

Beckmann, M. (1976). Spatial equilibrium in the dispersed city. En G. J. Papageorgiou (Ed.), Mathematical land use theory. Lexington: Lexington Books.

Borukhov, E., \& Hochman, O. (1977). Optimum and market equilibrium in a model of a city without a predetermined center. Environment and Planning A, 9(8), 849-856.

Cámara de Comercio de Bogotá. (2009). Perfil de la localidad de Puente Aranda. Bogotá: CGB.

DANE. (2009). Clasificación industrial internacional uniforme de todas las actividades económicas Rev. 3.1 Adaptado para Colombia. Bogotá: DANE. Recuperado de http://goo.gl/ cKdbiU

Jaramillo, S. (2006). Mercado del suelo urbano: precios, agentes, operaciones. Consideraciones teóricas para orientar la gestión de la tierra en las ciudades. Recuperado de http://goo.gl/XG8X2T

Karlqvist, A., \& Lundqvist, L. (1972). A contact model for spatial allocation. Regional Studies, 6(4), 401-419.

Lever, G. (2009). El modelo de precios hedónicos. Chile: Asociación de Arquitectos Tasadores de Chile. Recuperado de http://goo.gl/nScrnd

Papageorgiou, Y., \& Smith, T. (1983). Agglomeration as a local instability of uniform steady states. Econometrica, 51(4), 1109-1119.

Raco, M. (1997). Business associations and the politics of urban renewal: The case of the Lower Don Valley, Sheffield. Urban Studies, 34(3), 383-402.

Santagata, W., \& Ortona, G. (1981). Industrial mobility in the Turin metropolitan area, 1961-77. Urban Studies, 20(1), 59-71. 
ISSN: 0124-3551 / Año 16, No 25 / julio-diciembre / pp. 17-41

Sarzynski, A., Wolman, H. L., Galster, G., \& Hanson, R. (2006). Testing the conventional wisdom about land use and traffic congestion: The more we sprawl, the less we move? Urban Studies, 43(3), 601-626.

Shin, H. B. (2008). Living on the edge: financing post-displacement housing in urban redevelopment projects in Seoul. Environment and Urbanization, 20(2), 411-426.

Solow, R. M., \& Vickrey, W. (1971). Land use in a long narrow city. Fournal of Economic Theory, 3(4), 430-447.

Sullivan, A. (1986). A general equilibrium model with agglomerative economies and decentralized employment. Fournal of Urban Economics, 20(1), 55-74.

Usowski, K. G. (1994). Neoclassical analysis of monocentric and multicentric urban equilibria (Tesis doctoral). Northwestern University, Illinois.

Vickerman, R. W. (1979). The evaluation of urban change: Equilibrium and adaptive approaches. Urban Studies, 16(1), 81-93.

White, M. (1988). Location choice and commuting behavior in cities with decentralized employment. Fournal of Urban Economics, 24, 129-152. Recuperado de http://econweb.ucsd.edu/ miwhite/location-choice-commuting.pdf

Wieand, K. (1987). An extension of the monocentric urban spatial equilibrium model to a multicenter setting: The case of the two-center city. Fournal of Urban Economics, 21(3), 259-271.

Xie, Q., Parsa, A., \& Redding, B. (2002). The emergence of the urban land market in China: Evolution, structure, constraints and perspectives. Urban Studies, 39(8), 1375-1398.

Xie, Q. (1995). Policies for land prices in China. Journal of Central China Normal University, Special Issue on Real Estate, 171-175. 


\section{Anexos}

Especificación de modelos de datos panel: Cambios 2000 / 2010

La modelación se realizó utilizando el conocimiento del territorio a partir de algunas agrupaciones que se describirán posteriormente. Constituye un panel de datos, especificando la siguiente ecuación a estimar:

$$
y=f(d, u, a, p)
$$

donde

$y=$ avaluo

$d=$ densidad

$u=u s o$

$a=$ arealote

$p=$ tipopropiedad

La pretensión de este análisis es mostrar una referencia en la cual se pueda modelar, a partir de los efectos fijos o efectos aleatorios, una expresión del precio del suelo como variable que resume los gustos o preferencias idiosincráticas de los individuos, esto es, variable $u_{i j}$

\section{Caracterización de los registros catastrales}

Los cambios en la década 2000 / 2010. Utilizada para la determinación y estimación de los modelos de datos panel, se utilizaron las muestras comparativas de una década; corresponde a la información de la siguiente tabla:

Tabla 2. Puente Aranda: resumen principales variables cuantitativas 2000 / 2010

\begin{tabular}{|r|r|r|r|r|r|r|}
\hline Año & $\begin{array}{c}\text { Cantidad } \\
\text { de lotes }\end{array}$ & \multicolumn{1}{|c|}{$\begin{array}{c}\text { Área } \\
\text { terreno }\end{array}$} & $\begin{array}{c}\text { Valor } \\
\text { promedio } \mathrm{m}^{2} \\
\text { terreno }\end{array}$ & $\begin{array}{c}\text { Área } \\
\text { construida } \\
\mathrm{m}^{2}\end{array}$ & $\begin{array}{c}\text { Valor } \\
\text { promedio } \mathrm{m}^{2} \\
\text { construcción }\end{array}$ & $\begin{array}{c}\text { Valor promedio } \\
\text { total avalúo }\end{array}$ \\
\hline 2000 & 57.974 & 11.943 .909 & 209.423 & 12.074 .005 & 265.249 & 58.576 .660 \\
\hline 2010 & 64.840 & 13.273 .403 & 510.826 & 13.822 .482 & 410.166 & 140.214 .861 \\
\hline Crec $\%$ & $11,84 \%$ & $11,13 \%$ & $143,92 \%$ & $14,48 \%$ & $54,63 \%$ & $139,37 \%$ \\
\hline
\end{tabular}

Fuente: Registro catastral bases 2000 - 2010 
En la tabla se observa que, en correlación con un incremento de la cantidad de área de terreno total de la localidad, la cantidad de predios ha aumentado. Esto se debe a ajustes sobre la medición de las áreas de terreno realizados por la institucionalidad distrital. Si bien los límites de la localidad son los mismos, estos ajustes están enfocados a actualizaciones de la información específica de cada predio. Los efectos de la valorización en una década se evidencian con un crecimiento del valor promedio del metro cuadrado de terreno, aumento superior al crecimiento del valor promedio del metro cuadrado de construcción, marcando un crecimiento general del 139,4 \% del promedio de avalúo del precio total, que incluye el valor del terreno y el valor de las construcciones.

Para facilitar la estimación, se agruparon los destinos de uso del suelo ${ }^{12}$ y los tipos de propiedad ${ }^{13}$ en cuatro grupos o variables categóricas: residencial, industrial y comercial, dotacional y otros. Las agrupaciones de la tabla anterior se realizaron con el fin de facilitar la discriminación de los cambios en los usos a partir de los valores diferenciados entre estos códigos, como se observa en la siguiente tabla:

Tabla 3. Puente Aranda: resumen de códigos de cambio

\begin{tabular}{|cl|}
\hline Código de Cambio & \multicolumn{1}{c|}{ Tipo de Cambio } \\
\hline 0 & No hay Cambio \\
-3 & Residencial a dotacional \\
-1 & Residencial a industrial \\
-10 & Residencial a otros \\
\hline 10 & Otros a residencial \\
\hline 9 & Otros a industrial y comercial \\
\hline 7 & Otros a dotacional \\
1 & Industrial a residencial \\
-9 & Industrial a otros \\
-2 & Industrial a dotacional \\
-7 & Dotacional a otros \\
\hline 2 & Dotacional a industrial \\
3 & Dotacional a industrial \\
\hline
\end{tabular}

Fuente: Registro catastral bases 2000 - 2010. Agrupaciones del autor.

Se puede observar que el $73 \%$ de los predios no presentó cambio de uso en la década de análisis, los cambios de uso industrial y comercial a residencial y de residencial a industrial y comercial presentaron un comportamiento similar cercano al $12 \%$, los demás usos presentaron dinámicas inferiores al $1 \%$ de participación.

12 Los destinos corresponden a la orientación catastral que se le ha dado al suelo.

13 Corresponde a la caracterización de los dueños de cada lote de terreno. 
Determinantes del precio del suelo como variable proxy de las preferencias idiosincráticas de los individuos...

Así mismo, se observaron mayores dinámicas en las áreas de terreno construidas para los predios que cambiaron sus usos de industrial y comercial a residencial, lo que se observa es un fomento de la densificación para el uso residencial.

Tabla 4. Puente Aranda: resumen de cambios destinos 2000 / 2010

\begin{tabular}{|c|c|c|c|c|c|c|c|}
\hline 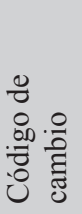 & 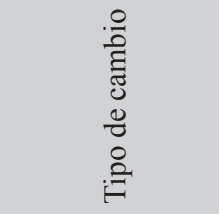 & 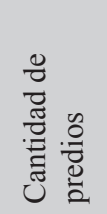 & 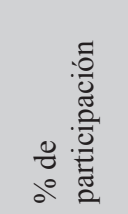 & 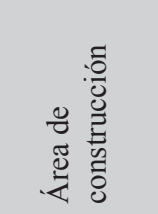 & 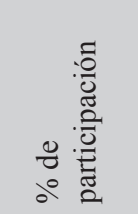 & 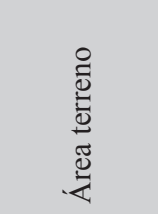 & 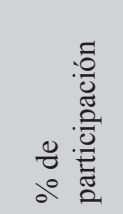 \\
\hline-10 & $\begin{array}{l}\text { Residencial a } \\
\text { otros }\end{array}$ & 44 & $0,08 \%$ & 6.909 & $0,05 \%$ & 4.315 & $0,04 \%$ \\
\hline-9 & $\begin{array}{l}\text { Industrial a } \\
\text { otros }\end{array}$ & 41 & $0,07 \%$ & 36.435 & $0,29 \%$ & 71.970 & $0,69 \%$ \\
\hline-3 & $\begin{array}{l}\text { Residencial a } \\
\text { dotacional }\end{array}$ & 538 & $0,95 \%$ & 92.911 & $0,74 \%$ & 53.314 & $0,51 \%$ \\
\hline-2 & $\begin{array}{l}\text { Industrial a } \\
\text { dotacional }\end{array}$ & 211 & $0,37 \%$ & 95.512 & $0,76 \%$ & 75.182 & $0,73 \%$ \\
\hline-1 & $\begin{array}{l}\text { Residencial a } \\
\text { industrial }\end{array}$ & 6.299 & $11,14 \%$ & 1.113 .121 & $8,83 \%$ & 629.719 & $6,08 \%$ \\
\hline 0 & No hay Cambio & 41.288 & $73,03 \%$ & 7.555 .880 & $59,94 \%$ & 4.951 .023 & $47,79 \%$ \\
\hline 1 & $\begin{array}{l}\text { Industrial a } \\
\text { residencial }\end{array}$ & 7.241 & $12,81 \%$ & 3.205 .549 & $25,43 \%$ & 2.726 .244 & $26,32 \%$ \\
\hline 2 & $\begin{array}{l}\text { Dotacional a } \\
\text { industrial }\end{array}$ & 101 & $0,18 \%$ & 252.783 & $2,01 \%$ & 473.014 & $4,57 \%$ \\
\hline 3 & $\begin{array}{l}\text { Dotacional a } \\
\text { residencial }\end{array}$ & 359 & $0,64 \%$ & 244.731 & $1,94 \%$ & 682.524 & $6,59 \%$ \\
\hline 7 & $\begin{array}{l}\text { Otros a } \\
\text { Dotacional }\end{array}$ & 11 & $0,02 \%$ & - & $0,00 \%$ & 38.630 & $0,37 \%$ \\
\hline 9 & $\begin{array}{l}\text { Otros a } \\
\text { Industrial y } \\
\text { Comercial }\end{array}$ & 91 & $0,16 \%$ & 490 & $0,00 \%$ & 120.883 & $1,17 \%$ \\
\hline 10 & $\begin{array}{l}\text { Otros a } \\
\text { Residencial }\end{array}$ & 309 & $0,55 \%$ & 1.263 & $0,01 \%$ & 532.730 & $5,14 \%$ \\
\hline To & de predios & 56.533 & $100,00 \%$ & 12.605 .584 & $100,00 \%$ & 10.359 .548 & $100,00 \%$ \\
\hline
\end{tabular}

Fuente: Registro catastral bases 2000 - 2010. Agrupaciones y cálculos del autor.

Bajo la misma metodología se han agrupado los tipos de propiedad en tres categorías: oficial, espacio público y particular, cuyo resumen se observa en la siguiente tabla: 
Tabla 5. Puente Aranda: resumen códigos cambios tipo propiedad 2000 / 2010

\begin{tabular}{|c|c|}
\hline Código cambio & \multicolumn{1}{c|}{ Tipo de Cambio } \\
\hline-3 & De particular a espacio publico \\
\hline-2 & De oficial a espacio publico \\
\hline-1 & De particular a oficial \\
\hline 0 & No hay cambio \\
\hline 1 & De oficial a particular \\
\hline 2 & De espacio publico a oficial \\
\hline 3 & De espacio publico a oficial \\
\hline
\end{tabular}

Fuente: registro catastral bases 2000 - 2010; Agrupaciones y cálculos del autor

El principal cambio observable en los tipos de propiedad son los predios que pasaron de dueños particulares a espacio público (5.89\% de participación), así mismo, el 93 \% de los predios mantuvo su situación de propiedad y los demás cambios fueron inferiores al $1 \%$ de participación.

Tabla 6. Puente Aranda: resumen de cambios propiedad 2000 / 2010

\begin{tabular}{|c|c|c|c|}
\hline Código cambio & \multicolumn{1}{|c|}{ Tipo de cambio } & Cantidad predios & \% participación \\
\hline-3 & De particular a espacio publico & 3.330 & $5,89 \%$ \\
\hline-2 & De oficial a espacio publico & 23 & $0,04 \%$ \\
\hline-1 & De particular a oficial & 153 & $0,27 \%$ \\
\hline 0 & No hay cambio & 53.582 & $93,01 \%$ \\
\hline 1 & De oficial a particular & 355 & $0,63 \%$ \\
\hline 2 & De espacio publico a oficial & 1 & $0,00 \%$ \\
\hline
\end{tabular}

Fuente: registro catastral bases 2000 - 2010. Agrupaciones y cálculos del autor.

Este comportamiento está relacionado con la teoría de la fijación de la renta de los terrenos ${ }^{14}$, según la cual, las transformaciones se dan derivadas de la búsqueda de ma-

14 Samuel Jaramillo: Consideraciones teóricas para orientar la gestión de la tierra en las ciudades, referenciado en World Bank. 
Determinantes del precio del suelo como variable proxy de las preferencias idiosincráticas de los individuos...

yores niveles de rentas al cambiar el uso dado, que existen unas rentas primarias diferenciales y rentas urbanas secundarias que corresponden a la renta diferencial de comercio, rentas industriales, renta de segregación, renta diferencial de vivienda, cuyas dinámicas se entrelazan y orientan la toma de decisiones de los dueños de una propiedad inmobiliaria.

\section{La productividad de las firmas, estática del año 2009:}

\section{Caracterización del Registro Mercantil}

Del Registro Mercantil disponible se observa que la actividad empresarial localizada en la zona tiene las siguientes características:

Evidencia que la mayor cantidad de firmas grandes se concentra en actividades de industria manufacturera $(52,6 \%)$, comercio $(25,43 \%)$ y actividades inmobiliarias, de alquiler y empresariales $(8,09 \%$ ). Suman el 86,12\% de las firmas grandes de la localidad.

La distribución es similar en la escala de las empresas medianas, con un 44,52 \% de empresas dedicadas a la industria manufacturera, 29,46\% a actividades de comercio, actividades inmobiliarias y de alquiler con 13,33\% y el transporte, almacenamiento y comunicaciones con un $4,52 \%$.

Frente a la escala de las pequeñas empresas y las microempresas, las actividades comerciales son las mayores en participación, con un 44,06 \%, seguidas por la industria manufacturera con un 24,51\% y las actividades inmobiliarias y de alquiler con un $8,06 \%$; los hoteles y restaurantes presentan gran vocación en la localidad, con un 6,24 \%, y el transporte, almacenamiento y comunicaciones con un 6,23\%. Estas dinámicas de pequeña escala de las actividades económicas se orientan a suplir demanda de la actividad industrial y comercial de mayor escala presente en la zona.

Tabla 7. Puente Aranda. Actividad económica 2009

\begin{tabular}{|c|l|c|c|}
\hline CIIU & \multicolumn{1}{|c|}{ Actividad económica } & No. Empresas & \% de participación \\
\hline A & Agricultura, ganadería, caza y silvicultura & 95 & $1,13 \%$ \\
\hline B & Pesca & 3 & $0,04 \%$ \\
\hline C & Explotación de minas y canteras & 21 & $0,25 \%$ \\
\hline D & Industrias Manufactureras & 2.173 & $25,80 \%$ \\
\hline E & Suministro de electricidad, gas y agua & 20 & $0,12 \%$ \\
\hline F & Construcción & 241 & $2,86 \%$ \\
\hline
\end{tabular}


ISSN: 0124-3551 / Año 16, No 25 / julio-diciembre / pp. 17-41

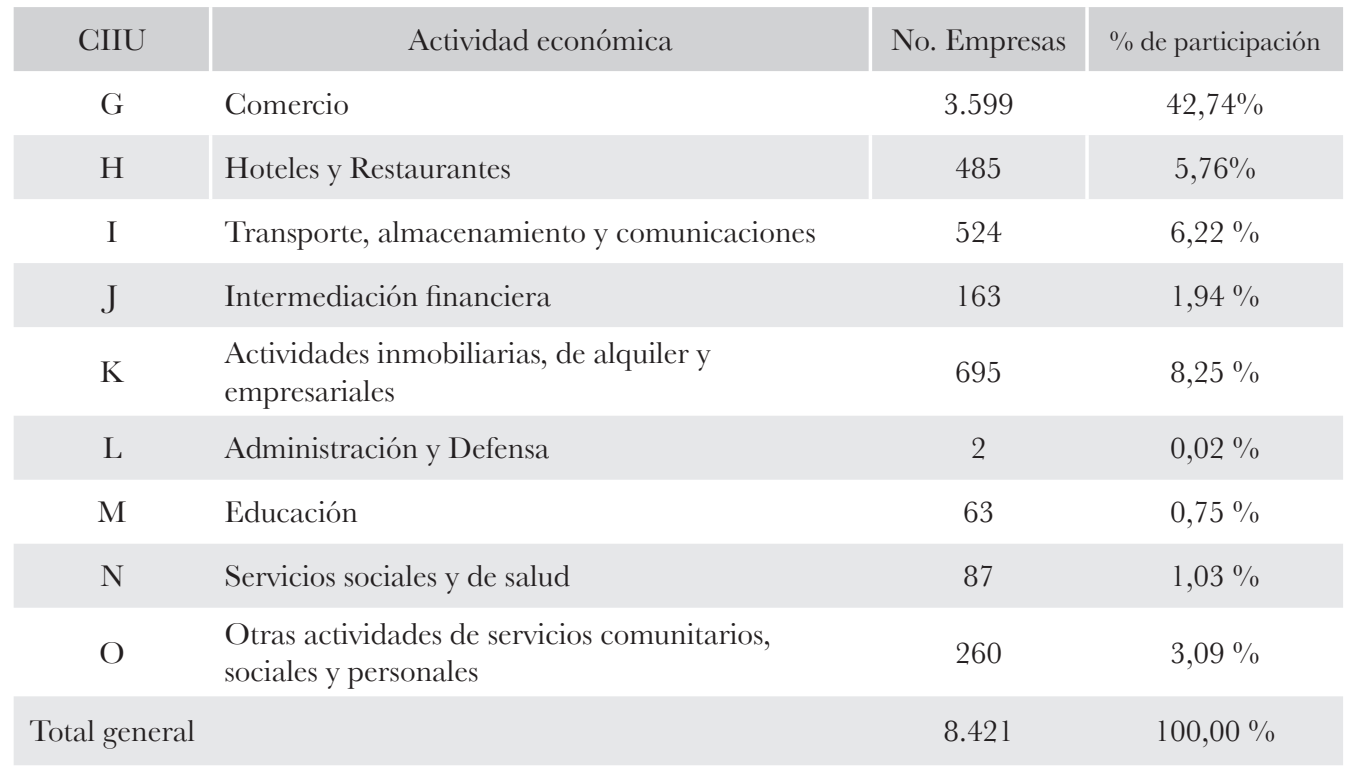

Fuente: registro mercantil CCB - Base 2009. Cálculos del autor.

La muestra utilizada en la presente investigación corresponde a un 3,56 \% de las empresas registradas en la Cámara de Comercio de Bogotá ${ }^{15}$.

Tabla 8. Puente Aranda. Tipos de empresa por tamaño 2009

\begin{tabular}{|c|c|c|}
\hline Tipo de empresa & no. de empresas & $\%$ de participación \\
\hline Grandes & 173 & $2,11 \%$ \\
\hline Medianas & 465 & $5,66 \%$ \\
\hline Microempresas & 6253 & $76.13 \%$ \\
\hline Pequeños & 1323 & $16,11 \%$ \\
\hline Total general & 8214 & $100,00 \%$ \\
\hline
\end{tabular}

Fuente: registro Mercantil CCB - Base 2009. Cálculos del autor.

La escala de las empresas determina la productividad y generación de empleo en un sector determinado; además, dadas las actividades económicas, que se traducen en tipos de tecnología, es posible enriquecer el siguiente Modelo de Fijación o determinación de precios al incluir la variable cualitativa de la actividad económica de acuerdo con las anteriores tablas.

15 Utilizando como soporte para el cálculo el dato proveniente de la base de datos general sin depuración de la CCB, en el cual se obtienen 228062 registros de empresas en las localidades de Bogotá. 
Determinantes del precio del suelo como variable proxy de las preferencias idiosincráticas de los individuos...

Jorge Andrés Talero Bernal

Tabla 9. Puente Aranda. Ventas, activos, personal y ganancias por tipo de empresa

\begin{tabular}{|c|c|c|c|c|c|}
\hline $\begin{array}{c}\text { Tipo de } \\
\text { empresa }\end{array}$ & $\begin{array}{l}\text { Cantidad de } \\
\text { Estableci- } \\
\text { mientos }\end{array}$ & Ventas & Activos & Personal & Ganancias \\
\hline Grandes & 173 & $\begin{array}{c}15.001 .249 .352 .69 \\
6,60\end{array}$ & $\begin{array}{c}7.140 .380 .822 .53 \\
5,19\end{array}$ & $48.794,00$ & $\begin{array}{c}508.723 .809 .02 \\
6,47\end{array}$ \\
\hline Medianas & 465 & $\begin{array}{c}3.163 .836 .726 .57 \\
1,82\end{array}$ & $\begin{array}{c}1.670 .930 .647 .14 \\
5,08\end{array}$ & $48.599,50$ & $\begin{array}{c}128.799 .760 .91 \\
6,04\end{array}$ \\
\hline Pequeños & $1.323,00$ & $\begin{array}{c}2.242 .633 .267 .65 \\
9,67\end{array}$ & $\begin{array}{c}870.552 .651 .05 \\
9,12\end{array}$ & $25.798,00$ & $\begin{array}{c}78.841 .192 .99 \\
2,48\end{array}$ \\
\hline Microempresas & $6.253,00$ & $\begin{array}{c}1.254 .361 .693 .24 \\
7,23\end{array}$ & $\begin{array}{c}177.024 .374 .78 \\
8,25\end{array}$ & $31.205,00$ & $\begin{array}{c}69.587 .692 .97 \\
5,55\end{array}$ \\
\hline Total Localidad & $8.214,00$ & $\begin{array}{c}21.688 .081 .040 .17 \\
5,4\end{array}$ & $\begin{array}{c}9.858 .888 .495 .52 \\
8,22\end{array}$ & $154.396,50$ & $\begin{array}{c}786.222 .455 .91 \\
0,58\end{array}$ \\
\hline
\end{tabular}

Fuente: registro catastral 2009 y registro mercantil 2009. Cálculos del autor.

En la tabla es posible resaltar la actividad económica de la localidad al contrastar el valor agregado de las ventas; estas representan alrededor del 17,6\% del PIB de la ciudad en el año de la investigación ${ }^{16}$.

Tabla 10. Puente Aranda. Productividad promedio del empleo ${ }^{17}$

\begin{tabular}{|l|c|c|}
\hline \multicolumn{1}{|c|}{ Tipo de empresa } & $\begin{array}{c}\text { Productividad promedio } \\
\text { empleo anual }\end{array}$ & $\begin{array}{c}\text { Productividad promedio } \\
\text { empleo mensual }\end{array}$ \\
\hline Grandes & $20.092 .166,61$ & $1.674 .347,22$ \\
\hline Medianas & $20.803 .090,62$ & $1.733 .590,89$ \\
\hline Pequeños & $2.468 .980,25$ & $205.748,35$ \\
\hline Microempresas & $4.956 .017,48$ & $413.001,45$ \\
\hline Promedio Localidad & $12.080 .063,74$ & $1.006 .671,98$ \\
\hline
\end{tabular}

Fuente: registro catastral 2009 y registro mercantil, 2009. Cálculos del autor.

16 Sobre un PIB corriente consolidado cercano a los 127 billones de pesos, estimado para el año 2009.

17 Calculada utilizando como referencia el promedio ponderado de cada uno de los establecimientos, siguiendo la formulación:

$\frac{\sum_{i=1}^{R} \text { GANANCLAEST, }}{\sum_{i=1}^{E} E S T_{e}}$ 


\section{Generación de empleo}

A continuación, se observan las dinámicas de generación de empleo por actividad económica ${ }^{18}$ y tamaño de empresa en la localidad, en las cuales se resume que las actividades económicas de mayor generación de empleo son las industrias manufactureras, el comercio, los hoteles y restaurantes, las actividades inmobiliarias, de alquiler y empresariales, y el transporte y almacenamiento.

Tabla 11. Puente Aranda. Empleo por tamaño de empresa

\begin{tabular}{|c|c|c|c|c|c|c|}
\hline CIIU & Actividad económica & Grandes & Medianas & Pequeñas & $\begin{array}{c}\text { Micro- } \\
\text { empresas }\end{array}$ & $\begin{array}{c}\text { Total } \\
\text { sector }\end{array}$ \\
\hline A & $\begin{array}{l}\text { Agricultura, ganadería, } \\
\text { caza y silvicultura }\end{array}$ & 2.297 & 752 & 392 & 252 & 3.692 \\
\hline B & Pesca & - & - & - & 11 & 11 \\
\hline $\mathrm{C}$ & $\begin{array}{l}\text { Explotación de minas y } \\
\text { canteras }\end{array}$ & - & 79 & 90 & 70 & 239 \\
\hline $\mathrm{D}$ & Industrias Manufactureras & 22.300 & 18.506 & 10.211 & 7.069 & 58.086 \\
\hline $\mathrm{E}$ & $\begin{array}{l}\text { Suministro de electricidad, } \\
\text { gas y agua }\end{array}$ & 207 & - & 20 & 35 & 262 \\
\hline $\mathrm{F}$ & Construcción & 220 & 664 & 854 & 910 & 2.647 \\
\hline G & Comercio & 13.314 & 10.019 & 7.430 & 13.899 & 44.661 \\
\hline $\mathrm{H}$ & Hoteles y Restaurantes & 1.660 & 232 & 158 & 2.287 & 4.337 \\
\hline I & $\begin{array}{l}\text { Transporte, } \\
\text { almacenamiento y } \\
\text { comunicaciones }\end{array}$ & 402 & 2.612 & 1.731 & 1.927 & 6.672 \\
\hline $\mathrm{J}$ & Intermediación financiera & 1.012 & 925 & 341 & 354 & 2.632 \\
\hline $\mathrm{K}$ & $\begin{array}{l}\text { Actividades inmobiliarias, } \\
\text { de alquiler y empresariales }\end{array}$ & 5.037 & 14.444 & 3.969 & 2.493 & 25.943 \\
\hline $\mathrm{L}$ & Administración y Defensa & - & - & 8 & - & 8 \\
\hline $\mathrm{M}$ & Educación & - & - & 76 & 301 & 377 \\
\hline $\mathrm{N}$ & Servicios sociales y de salud & - & 116 & 144 & 379 & 638 \\
\hline $\mathrm{O}$ & $\begin{array}{l}\text { Otras actividades de } \\
\text { servicios comunitarios, } \\
\text { sociales y personales }\end{array}$ & 2.354 & 253 & 377 & 1.218 & 4.193 \\
\hline \multicolumn{2}{|c|}{ Total general } & 48.794 & 48.600 & 25.798 & 31.205 & 154.397 \\
\hline
\end{tabular}

Fuente: registro catastral 2009 y registro mercantil, 2009. Cálculos del autor.

18 Utilizando como base la Clasificación industrial internacional uniforme de todas las actividades económicas Rev 3.1 adaptado para Colombia, DANE, 2009. 
Determinantes del precio del suelo como variable proxy de las preferencias idiosincráticas de los individuos...

Jorge Andrés Talero Bernal

Tabla 12. Puente Aranda. Empleo, ventas y ganancias por actividad económica 2009

\begin{tabular}{|c|c|c|c|c|}
\hline CIIU & Actividad económica & Personal & Ventas & Ganancias \\
\hline A & $\begin{array}{l}\text { Agricultura, ganadería, caza } \\
\text { y silvicultura }\end{array}$ & 3.692 & $1.030 .602 .725 .997,40$ & $22.460 .034 .669,22$ \\
\hline $\mathrm{B}$ & Pesca & 11 & $2.819 .673 .164,32$ & 24.233 .763 \\
\hline $\mathrm{C}$ & $\begin{array}{l}\text { Explotación de minas y } \\
\text { canteras }\end{array}$ & 239 & $15.185 .697 .024,76$ & $822.852 .589,17$ \\
\hline $\mathrm{D}$ & Industrias Manufactureras & 58.086 & $9.473 .894 .009 .871,68$ & 178.983.130.220,06 \\
\hline $\mathrm{E}$ & $\begin{array}{l}\text { Suministro de electricidad, } \\
\text { gas y agua }\end{array}$ & 262 & 116.659.228.117,99 & $2.106 .395 .072,28$ \\
\hline $\mathrm{F}$ & Construcción & 2.647 & 205.398.405.506,35 & 7.154.408.369,01 \\
\hline G & Comercio & 44.661 & 8.596.041.019.939,63 & $439.098 .985 .882,63$ \\
\hline $\mathrm{H}$ & Hoteles y Restaurantes & 4.337 & $182.847 .298 .738,16$ & 10.947.707.638,36 \\
\hline I & $\begin{array}{l}\text { Transporte, almacenamiento } \\
\text { y comunicaciones }\end{array}$ & 6.672 & $719.380 .866 .627,03$ & $26.301 .286 .402,94$ \\
\hline $\mathrm{J}$ & Intermediación financiera & 2.631 & $309.038 .216 .953,32$ & $10.996 .691 .208,53$ \\
\hline $\mathrm{K}$ & $\begin{array}{l}\text { Actividades inmobiliarias, de } \\
\text { alquiler y empresariales }\end{array}$ & 25.942 & $747.040 .999 .090,29$ & $72.271 .634 .393,14$ \\
\hline $\mathrm{L}$ & Administración y Defensa & 8 & $19.190 .048,00$ & $59.592 .738,47$ \\
\hline M & Educación & 377 & 13.988.989.308,68 & $599.416 .974,58$ \\
\hline $\mathrm{N}$ & Servicios sociales y de salud & 638 & $31.356 .023 .364,73$ & $1.832 .553 .392,73$ \\
\hline $\mathrm{O}$ & $\begin{array}{l}\text { Otras actividades de } \\
\text { servicios comunitarios, } \\
\text { sociales y personales }\end{array}$ & 4.193 & 223.808.696.421,95 & $12.563 .532 .622,69$ \\
\hline \multicolumn{2}{|c|}{ Total general } & 154.396 & $21.668 .081 .040 .174,30$ & $786.222 .455 .910,57$ \\
\hline
\end{tabular}

Fuente: registro catastral 2009 y registro mercantil, 2009. Cálculos del autor.

La gran contribución a la producción de la localidad es realizada por el sector industrias manufactureras $(43,72 \%)$, comercio, hoteles y restaurantes $(40,52 \%)$, y aportan a la generación de ganancias en un 57,2 4\% el comercio, el sector hoteles y restaurantes, el $22,76 \%$ industrias manufactureras, y el 9,19 \% es contribución de las actividades inmobiliarias, de alquiler y empresariales. 
